\title{
Neurodegenerative diseases-Caps: a capsule network based early screening system for the classification of neurodegenerative diseases
}

\author{
Kirti Raj Bhatele ${ }^{1} \cdot$ Anand Jha ${ }^{1} \cdot$ Kavish Kapoor $^{2} \cdot$ Devanshu Tiwari $^{3}$
}

Received: 13 September 2021 / Revised: 16 December 2021 / Accepted: 21 January 2022 / Published online: 9 February 2022

(C) The Author(s), under exclusive licence to Springer Nature B.V. 2022

\begin{abstract}
The two most generally diagnosed Neurodegenerative diseases are the Alzheimer and Parkinson diseases. So this paper presents a fully automated early screening system based on the Capsule network for the classification of these two Neurodegenerative diseases. In this study, we hypothesized that the Neurodegenerative diseases-Caps system based on the Capsule network architecture accurately performs the multiclass i.e. three class classification into either the Alzheimer class or Parkinson class or Healthy control and delivers better results in comparison other deep transfer learning models. The real motivation behind choosing the capsule network architecture is its more resilient nature towards the affine transformations as well as rotational \& translational invariance, which commonly persists in the medical image datasets. Apart from this, the capsule networks overcomes the pooling layers related deficiencies from which conventional CNNs are mostly affected and unable to delivers accurate results especially in the tasks related to image classification. The various Computer aided systems based on machine learning for the classification of brain tumors and other types of cancers are already available. Whereas for the classification of Neurodegenerative diseases, the amount of research done is very limited and the number of persons suffering from this type of diseases are increasing especially in developing countries like India, China etc. So there is a need to develop an early screening system for the correct multiclass classification into Alzheimer's, Parkinson's and Normal or Healthy control cases. The Alzheimer disease and Parkinson progression (ADPP) dataset is used in this research study for the training of the proposed Neurodegenerative diseases-Caps system. This ADPP dataset is developed with the aid of both the Parkinson's Progression Markers Initiative (PPMI) and Alzheimer's disease Neuroimaging Initiative (ADNI) databases. There is no such early screening system exist yet, which can perform the accurate classification of these two Neurodegenerative diseases. For the sake of genuine comparison, other popular deep transfer learning models like VGG19, VGG16, ResNet50 and InceptionV3 are implemented and also trained over the same ADPP dataset. The proposed Neurodegenerative diseases-Caps system deliver accuracies of 97.81, 98, 96.81\% for the Alzheimer, Parkinson and Healthy control or Normal cases with 70/30 (training/validation split) and performs way better as compare to the other popular Deep transfer learning models.
\end{abstract}

Keywords Neurodegenerative diseases · Parkinson - Alzheimer - Capsule network - ResNet50 • VGG 16 . VGG $19 \cdot$ InceptionV3 etc.

Kirti Raj Bhatele

kirtirajbhatele8@gmail.com

Anand Jha

mail2anandjha@yahoo.co.in

Kavish Kapoor

kavishkapoor97@gmail.com

Devanshu Tiwari

devanshu.tiwari28@gmail.com
RJIT, BSF Academy, Tekanpur, Gwalior, India

2 Kavlabs, Gwalior, India

3 RGPV, Bhopal, Madhya Pradesh, India 


\section{Introduction}

The Parkinson disease (PD) and Alzheimer's disease (AD) are the two most common diagnosed Neurodegenerative diseases, which generally diagnosed in old age people (Alzheimer's Association 2017). The common symptoms of a person suffering from Alzheimer are memory loss, difficulty in completing normal tasks, challenges in planning etc. The lessening of certain specific nerve cells alongside plaques (neuritic) just outside the neuron and accumulation of neurofibrillary tangles in the inner side of neuron (McKhann et al. 1984) is the major cause for the Alzheimer disease and disturbing the normal functionality of brain cells. The early diagnosis of $\mathrm{AD}$ will be very helpful for the proper care of patients as this $\mathrm{AD}$ is incurable. The most effective way of diagnosing the $\mathrm{AD}$ is the use of various medical imaging modalities like Positron emission tomography (PET), Computed tomography (CT), Magnetic resonance imaging (MRI) etc. to carry out the patient's brain scans. Since the James Parkinson was the first one to explain this Parkinson's disease so it is known as the Parkinson's disease. This PD is also commonly diagnosed in old age people. The slow movements, face with no expressions, difficulty in standing from the chair, muscle rigidity, tremors, shaky handwriting, very low speech etc. are its major symptoms (Borek et al. 2006). The deficiency of Dopamine (acts as a neurotransmitter) in the human brain is the major cause of PD, which occurs due to the depletion of neurons (Ferreri et al. 2006). The PD just like $\mathrm{AD}$ is also highly progressive in nature and at the same time incurable too. So its diagnosis in the early stages will be very helpful in terms of providing proper healthcare to patients. The MRIs scans are mostly preferred by the physicians as compare to other medical imaging modalities like CT, PET etc. for the diagnosis of PD because the accurate Neuro-anatomic biomarkers are exhibited by the MRIs scans. Even the radiologists find it very tough to examine and perceive the inherent details of subcortical structures taken in these MRI scans with the help of bare eye because of the three dimensional nature. So there is an imperative need of an early screening system which can assist the radiologist and perform the analysis at the same time easily handle the minute intrinsic details of these three dimensional brain structures (Bakator and Radosav 2018; Lundervold and Lundervold 2019). The general set up for acquiring the MRI images of patients suffering from Alzheimer and Parkinson is presented with the aid of Fig. 1 below.

The application of machine and deep learning in the context of classification of various brain structural disorders is proved to be very useful and research oriented especially in the last five years. The CAD systems based on machine learning utilizing the medical imaging data and electronic medical records are proved to be very accurate in terms of accurate classification as well as prediction of various brain disorders. Apart from this substantial amount of research is previously been carried out in order to propose numerous CAD systems for automating the task of diagnosis of liver cancer, breast cancer, lung cancers etc. utilizing the Structural MRI scans, fMRI, PET, CT etc. But still the magnitude of research done in the domain of Neurodegenerative disease classification is far less as compare to Glioma and other types of brain tumor detection. The Fig. 2 below simply presents a statistical comparison among the number of research articles and studies published in the domain of Brain tumor (Glioma) classification and Neurodegenerative diseases i.e. Alzheimer, Parkinson based on machine and deep learning.

The conventional neural networks and deep transfer learning models are very popular and widely used by the researchers because of their high performance and efficient nature. As in these neural networks and deep transfer learning models, there is no need of manual segmentation and feature extraction unlike conventional machine learning models, so these deep learning models are more popular and hence completely automate the task of either binary or multi class classification (Russakovsky et al. 2015). But these conventional neural networks also tends to suffered from some deficiencies especially related to pooling layers, which affects their performance especially in the image classification task. So in order to overcome the deficiencies of existing neural networks and deep transfer learning models, the Capsule networks are used especially for the small size, medium size and augmented image datasets. The use of Capsule network offers advantages like robustness towards data rotations and affine transformation, also requires small size training datasets etc. (Sabour et al. 2017). Another important attribute of Capsule network is the use of routing by agreement or dynamic routing in order to overcome the issues related to pooling layers (Sara et al. 2017; Worrall et al. 2017). The application of the Capsule network based models are already being tried out especially in the field of Covid-19 classification utilizing the Chest X ray and CT scans images (Afshar et al. 2020; Tiwari and Jain 2021; Heidarian et al. 2021) and has delivers very encouraging results. The Capsule network based models has also delivered very good results in the domain of breast cancer classification (Wang et al. 2021), Lung Cancer classification (Ali and Ali 2021; Adu et al. 2021a, b),Brain tumor classification (Adu et al. 2021a, b) and other cancer type classification. The Capsule network performs better for the image classification tasks as it tends to learn the good weights for feature extraction and image classification along with how to infer spatial pose parameters from the image (Jiménez-Sánchez et al. 2018). All 


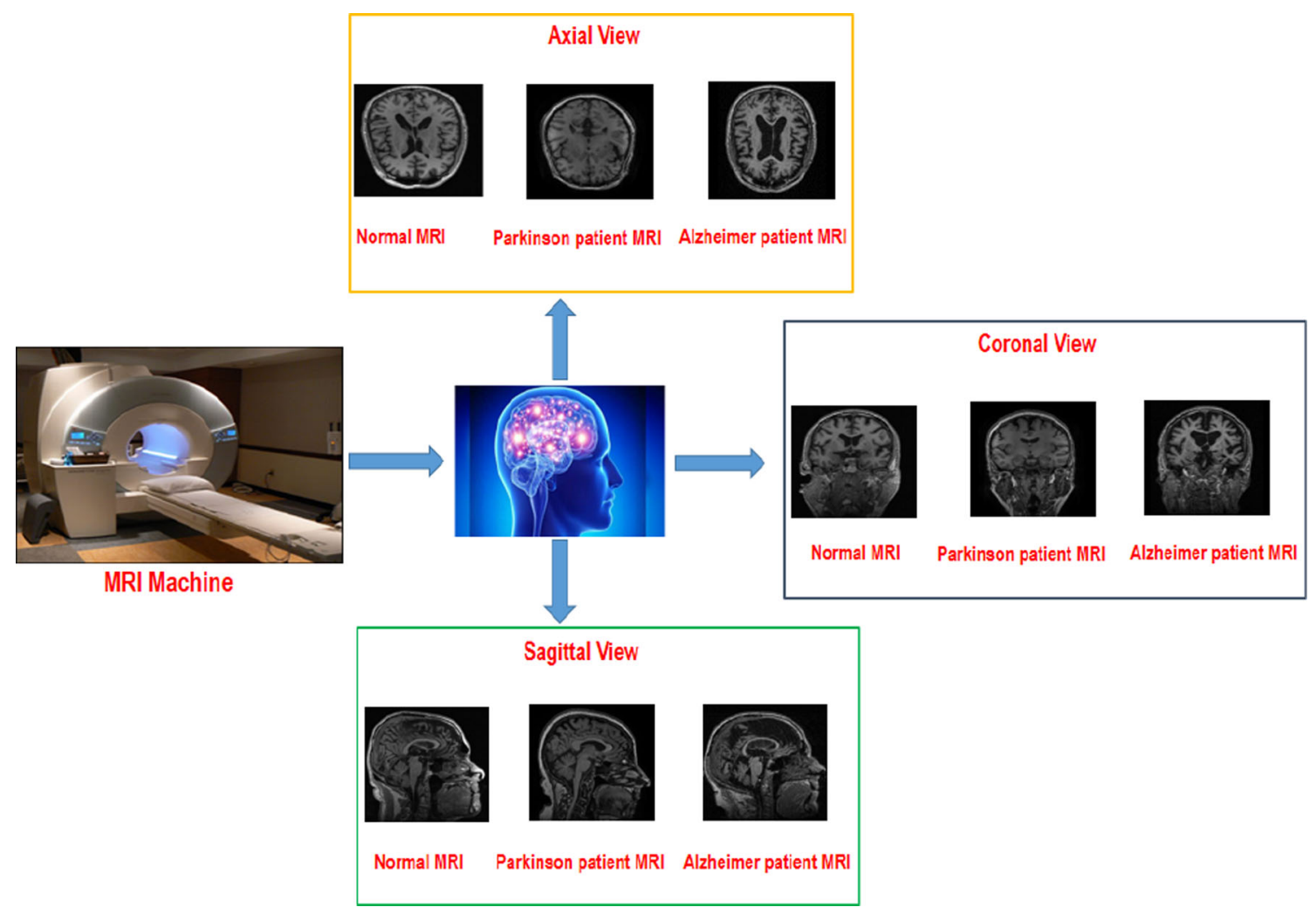

Fig. 1 General Procedure to acquire the MRI sequences from the patients suffering from Alzheimer and Parkinson diseases

Fig. 2 The yearly rise in statistics related to the amount of research studies associated with the use of artificial intelligence for the classification of Alzheimer, Parkinson and Brain tumor as per IEEE Xplore and PubMed databases from 2015 to 2020

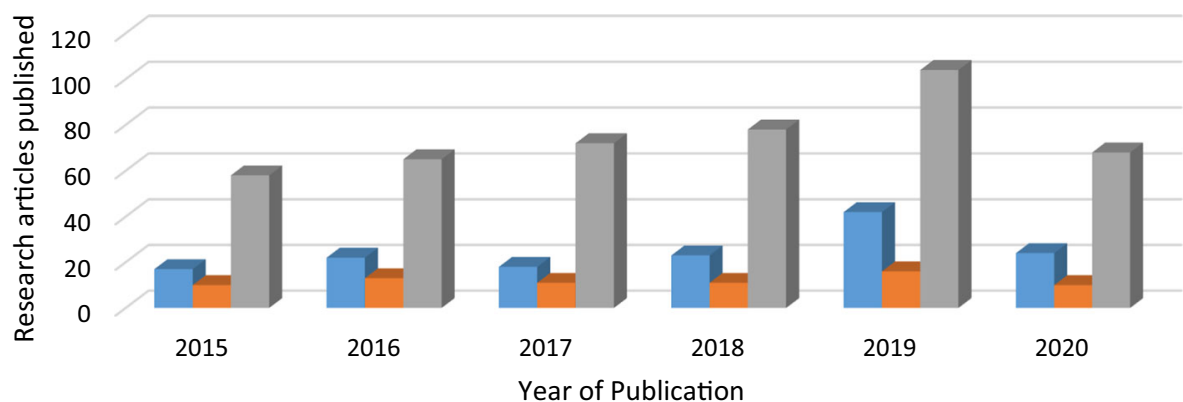

Machine learning based Alzeihmer classification Machine learning based Parkinson classification

Machine learning based Brain tumor classification these attributes makes the Capsule network an ideal alternative for the classification of multiclass Neurodegenerative disease classification. The major contributions of this research study are as follows:

- A Capsule network based framework was proposed for the Multiclass classification of Neurodegenerative diseases for the first time.

- In order to showcase the robustness, efficiency and applicability of this Neurodegenerative diseases-Caps system in real time, large size ADPP dataset is used for the training and testing purpose.
- To study the behavior and performance of our proposed Neurodegenerative diseases-Caps system in comparison with other popular deep transfer learning models. The VGG-16, VGG-19, ResNet 50 and InceptionV3 are also implemented and trained over the same ADPP dataset.

\section{Related work}

The magnitude of research done in the domain of Alzheimer and Parkinson disease classification using Machine and Deep learning is very limited. Initially the Alzheimer 
and Parkinson classification approaches based on Machine learning uses the classifiers like Support vector machine (SVM), Radial based function SVM (RBFS), least absolute shrinkage and selection operator (LASSO) etc. ( $\mathrm{Li}$ et al. 2014a, b; Dyrba et al. 2015; Garali et al. 2018; Peng et al. 2017; Amoroso et al. 2018). These classifiers are trained with the help of Diffusion tensor images (DTI) features, Graph theoretical measures, Entropy features, shape based and volume based features etc., which are extracted from the segmented region of interest. These machine learning based approaches in turn tends to perform either binary classification or multiclass classification categorizing the Alzheimer or Parkinson cases into their corresponding stages.

With the advent of Convolutional neural networks and Deep learning, the fully automated approaches for the binary as well as multiclass classification of Alzheimer and Parkinson diseases into their corresponding stages are proposed. Generally the Deep Convolutional Neural Network, Max Pooling based CNN, 3D neural network, enhanced probabilistic neural network framework (EPNN) etc. models are used in those approaches (Fulton et al. 2019; Talo et al. 2019; Yagis et al. 2019; Ramzan et al. 2020; Naz et al. 2021; Mozhdehfarahbakhsh et al. 2021). Majority of these Alzheimer and Parkinson classification approaches are utilizing the Global datasets like ADNI, PPMI, MIRIAD and OASIS etc. for the training and testing purpose. These datasets are having the Axial and Sagittal view MRI sequences. A brief comparison among some of the state of the art Alzheimer and Parkinson classification approaches based on the Machine as well as Deep learning are presented with the help of Tables 1 and 2 below.

The following points can be concluded from the brief literature review done above, which are as follows:

1. There is no unified system proposed yet either based on the Machine learning or deep learning for early screening of both the neurodegenerative diseases like Alzheimer and Parkinson. There is a need of a unified automated system in order to classify both the Alzheimer and Parkinson disease as both are neurodegenerative diseases in real time.

2. The majority of approaches for the classification of Alzheimer and Parkinson diseases are utilizing only the axial MRI scans, whereas there is still experimentation is required to be done with the sagittal view MRI scans and can even generate more accurate results. As these sagittal view MRI scans are mostly used by the radiologists for the diagnosis of Neurodegenerative diseases as they present more information about the grey matter content of the human brain.

3. As ADNI and PPMI databases are the mostly used repositories consist of MRI images of both the neurodegenerative diseases. So these two databases are used to develop a common ADPP datasets consisting of sagittal view Alzheimer diseases and Parkinson Progression MRI scans.

4. As Capsule networks are the evolving fields of deep neural networks delivering better results in present scenario. So the Capsule network can be utilized for the development of the Neurodegenerative disease as no such approach exist yet.

\section{Material and methods}

There are two major stages in the proposed Neurodegenerative diseases-Caps system. The initial stage is all about downloading the Sagittal view MRI scans of Alzheimer and Parkinson's diseases along with the healthy cases from the ADNI and PPMI global databases. As these MRI scans are present in the DICOM (Digital Imaging and Communications in Medicine) format, so convert them into the PNG (Portable Network Graphics) format as it offers compression of lossless nature. Followed by the proper selection of informative and precise sagittal view MRI scans with the aid of the Radiologists in order to develop training and testing datasets. The fine tuning and training of the Capsule Network with the help of this multiclass ADPP dataset is done in the second stage in order to perform the multiclass classification of Neurodegenerative dieses into Alzheimer or Parkinson or Healthy cases. The overall proposed Neurodegenerative disease-Caps system is illustrated with the help of Fig. 3 given below.

\section{ADPP dataset}

This dataset consist four thousand and eight hundred MRI scans of patients suffering from the Alzheimer and Parkinson diseases along with the scans of healthy controls i.e. normal cases for the training and validation purpose. From the ADNI and PPMI databases, around sixteen hundred Sagittal view MRI scans each of Alzheimer patients, Parkinson's patients and healthy controls are taken for training purpose. Almost all the researchers working in the domain of research are using these two ADNI (https://www.adni.loni.usc.edu) and PPMI (Marek et al. 2011) datasets. From the PPMI and ADNI databases around 90 healthy control cases, 80 Alzheimer and 80 Parkinson are downloaded in DICOM format. Each downloaded case will results into around 188-190 sagittal images, when converted from the DICOM format into the PNG format. Now out of those 188-190 sagittal images per case or subject around 20 sagittal images are selected with the help of Radiologist. In case of Alzheimer patient, the 
Table 1 A comparison among some of the state of the art Alzheimer disease classification approaches based on the Machine and Deep learning

\begin{tabular}{|c|c|c|c|c|}
\hline $\begin{array}{l}\text { Author } \\
\text { name and } \\
\text { year }\end{array}$ & $\begin{array}{l}\text { Machine learning classifier or Deep learning } \\
\text { model }\end{array}$ & Modalities used & $\begin{array}{l}\text { Result (Accuracy) } \\
\text { AD vs. healthy } \\
\text { control }(\%)\end{array}$ & $\begin{array}{l}\text { Datasets } \\
\text { used }\end{array}$ \\
\hline $\begin{array}{l}\text { Li et al. } \\
\quad(2014 a)\end{array}$ & LBP and SVM classifier & MRI sequence & 82.8 & $\begin{array}{l}\text { ADNI } \\
\text { database }\end{array}$ \\
\hline $\begin{array}{l}\text { Li et al. } \\
\quad(2014 b)\end{array}$ & SVM and Diffusion tensor images (DTI) features & T1 sequence MRI & 94.3 & $\begin{array}{l}\text { Local } \\
\text { dataset }\end{array}$ \\
\hline $\begin{array}{l}\text { Dyrba et al. } \\
\text { (2015) }\end{array}$ & SVM & sMRI, dMRI, Resting state-fMRI & 85 & $\begin{array}{l}\text { Local } \\
\text { dataset }\end{array}$ \\
\hline $\begin{array}{l}\text { Farzan } \\
\text { et al. } \\
(2015)\end{array}$ & RBFS & MRI sequence & 91.7 & ADNI \\
\hline $\begin{array}{r}\text { Ni et al. } \\
(2016)\end{array}$ & SVM and multiple kernel learning (MKL) & Resting state-fMRI & 76 & ADNI \\
\hline $\begin{array}{l}\text { Doan et al. } \\
\text { (2017) }\end{array}$ & LASSO & T1 sequence MRI & 81 & $\begin{array}{l}\text { Local } \\
\text { dataset }\end{array}$ \\
\hline $\begin{array}{l}\text { Glozman } \\
\text { et al. } \\
\text { (2017) }\end{array}$ & SVM & PET and MRI & 88.13 & ADNI \\
\hline $\begin{array}{l}\text { Guo et al. } \\
\text { (2017) }\end{array}$ & Multi-kernel SVM & Resting state-fMRI & 91.60 & $\begin{array}{l}\text { Local } \\
\text { dataset }\end{array}$ \\
\hline $\begin{array}{l}\text { Garali et al. } \\
\text { (2018) }\end{array}$ & SVM & FDG-PET & 97.88 & $\begin{array}{l}\text { Local } \\
\text { dataset }\end{array}$ \\
\hline $\begin{array}{l}\text { Wang et al. } \\
(2018)\end{array}$ & $\begin{array}{l}\text { The leaky rectified linear unit and max pooling } \\
\text { based CNN model }\end{array}$ & $\begin{array}{l}\text { T1-weighted Gradient-Echo sequence and } \\
\text { Magnetization-Prepared Rapid Gradient- } \\
\text { Echo (MP-RAGE) }\end{array}$ & 97.7 & $\begin{array}{l}\text { Local } \\
\text { dataset }\end{array}$ \\
\hline $\begin{array}{l}\text { Choi and } \\
\text { Jin }(2018)\end{array}$ & Deep convolutional neural network & PET images & 96 & ADNI \\
\hline $\begin{array}{l}\text { Goceri } \\
(2019)\end{array}$ & 3D neural network & MRI images & 98.06 & ADNI \\
\hline $\begin{array}{l}\text { Fulton et al. } \\
\text { (2019) }\end{array}$ & Resnet-50 & MRI images & 98 & OASIS \\
\hline $\begin{array}{l}\text { Talo et al. } \\
2019\end{array}$ & Resnet-34 & MRI images & 99 & $\begin{array}{l}\text { Local } \\
\text { dataset }\end{array}$ \\
\hline $\begin{array}{l}\text { El-Sappagh } \\
\text { et al. } \\
(2020)\end{array}$ & $\begin{array}{l}\text { Stacked convolutional neural network (CNN) } \\
\text { and Bidirectional long short-term memory } \\
\text { (BiLSTM) }\end{array}$ & MRI images & 92 & ADNI \\
\hline $\begin{array}{l}\text { Feng et al. } \\
\quad(2020)\end{array}$ & 3D-CNN-SVM & MRI images & 92 & ADNI \\
\hline $\begin{array}{l}\text { Ramzan } \\
\text { et al. } \\
(2020)\end{array}$ & ResNet18 & rs-fMRI & 97 & ADNI \\
\hline $\begin{array}{l}\text { Naz et al. } \\
\text { (2021) }\end{array}$ & $\begin{array}{l}\text { VGGNet, AlexNet, GoogLeNet, ResNet, } \\
\text { DenseNet, Inceptionv3, InceptionResNet }\end{array}$ & MRI images & $\begin{array}{l}97 \text { delivered by } \\
\text { VGGNet }\end{array}$ & ADNI \\
\hline
\end{tabular}

sagittal images that presents the best view of regions like entorhinal cortex, cerebral cortex and hippocampus are selected as these regions are precisely diagnosed by the radiologist for the correct prognosis of Alzheimer diseases. Whereas the sagittal images that best represents the Striatum, Substantia Nigra and Nigrostriatal regions of human brain are selected for the Parkinson disease cases as these regions are mostly affected in a patient suffering from the Parkinson disease. Similarly the sagittal images that best presents the above mentioned regions of human brain are selected in case of healthy control. The selected images undergoes preprocessing in order to remove the image artefacts. The images free from all type of artefacts are now used for the training and testing purpose. Apart from this a separate ADPP testing dataset is also used consist of only 200 sagittal images of Alzheimer, Parkinson and Normal cases each. 
Table 2 A comparison among some of the state of the art Parkinson disease classification approaches based on the Machine and Deep learning

\begin{tabular}{|c|c|c|c|c|}
\hline Author name and year & $\begin{array}{l}\text { Machine learning classifier or deep } \\
\text { learning model }\end{array}$ & Modalities used & $\begin{array}{l}\text { Result (accuracy) PD } \\
\text { vs. healthy control (\%) }\end{array}$ & $\begin{array}{l}\text { Datasets } \\
\text { used }\end{array}$ \\
\hline Adeli et al. (2016) & $\begin{array}{l}\text { Least squares formulation of linear } \\
\text { discriminant analysis (LS-LDA) } \\
\text { classifier }\end{array}$ & T1 sequence MRI & 82 & PPMI \\
\hline Peng et al. (2017) & $\begin{array}{l}\text { Multi-kernel support vector machine } \\
\text { (SVM) }\end{array}$ & T1 weighted MRI & 86 & PPMI \\
\hline Amoroso et al. (2018) & Random forest and SVM classifiers & T1 weighted MRI & 93 & PPMI \\
\hline Oliveira et al. (2017) & SVM & $\begin{array}{l}\text { The single-photon emission computed } \\
\text { tomography scans (FP-CIT SPECT) }\end{array}$ & 98 & PPMI \\
\hline Yagis et al. (2019) & VGG 16 and ResNet 50 & T1 weighted MRI & 82 & PPMI \\
\hline $\begin{array}{l}\text { Chakraborty et al. } \\
\text { (2020) }\end{array}$ & $\begin{array}{l}\text { 3D convolutional neural network }(\mathrm{CNN}) \\
\text { architecture }\end{array}$ & T1 weighted MRI & 95 & PPMI \\
\hline Vyas et al. (2021) & $2 \mathrm{D}$ and $3 \mathrm{D} \mathrm{CNN}$ & T1 weighted MRI & 88.9 & PPMI \\
\hline $\begin{array}{l}\text { Mozhdehfarahbakhsh } \\
\text { et al. (2021) }\end{array}$ & Customize CNN & T1 weighted MRI & 94 & PPMI \\
\hline
\end{tabular}

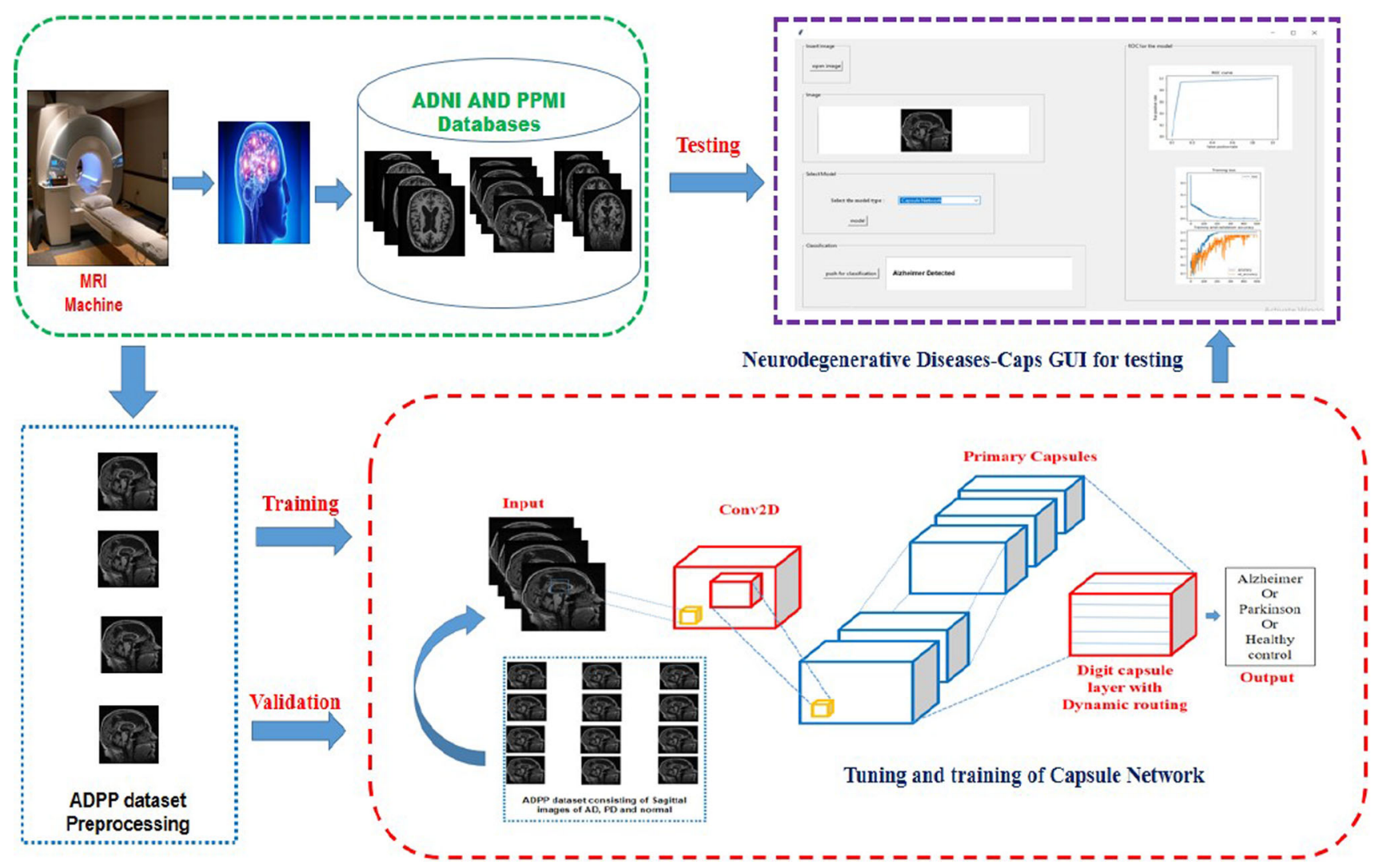

Fig. 3 The proposed neurodegenerative disease-Caps system architecture for the multiclass classification of Neurodegenerative diseases

The Table 3 below simply illustrates the demographic information of the ADPP dataset used in this paper.

\section{Capsule network}

Capsule network comprises of capsules. In capsule network each capsule is a group of neurons. Activity vectors of the neurons of a capsule represents different instantiation 
Table 3 The Demographic information of the ADPP dataset

\begin{tabular}{|c|c|c|c|c|c|c|c|c|}
\hline \multirow[t]{2}{*}{$\begin{array}{l}\text { Global } \\
\text { dataset }\end{array}$} & \multirow[t]{2}{*}{ Type } & \multirow[t]{2}{*}{$\begin{array}{l}\text { No. of cases taken for } \\
\text { training }\end{array}$} & \multirow[t]{2}{*}{$\begin{array}{l}\text { No. of cases taken for } \\
\text { testing }\end{array}$} & \multirow[t]{2}{*}{ Gender } & \multirow[t]{2}{*}{$\begin{array}{l}\text { Cases age } \\
\text { group }\end{array}$} & \multirow[t]{2}{*}{$\begin{array}{l}\text { MRI Modality } \\
\text { view }\end{array}$} & \multicolumn{2}{|c|}{$\begin{array}{l}\text { Number of scans } \\
\text { taken for }\end{array}$} \\
\hline & & & & & & & Training & Testing \\
\hline \multirow[t]{2}{*}{ ADNI } & $\mathrm{AD}$ & 80 & 14 & $\begin{array}{l}24 \text { females and } 26 \\
\text { males }\end{array}$ & $65-90$ & Sagittal & 1600 & 200 \\
\hline & $\mathrm{HC}$ & 50 & 8 & $\begin{array}{l}35 \text { females and } 15 \\
\text { males }\end{array}$ & $60-93$ & Sagittal & 900 & 100 \\
\hline \multirow[t]{2}{*}{ PPMI } & PD & 80 & 14 & $\begin{array}{l}20 \text { females and } 30 \\
\text { males }\end{array}$ & $39-80$ & Sagittal & 1600 & 200 \\
\hline & $\mathrm{HC}$ & 40 & 8 & $\begin{array}{l}26 \text { females and } 24 \\
\text { males }\end{array}$ & $31-80$ & Sagittal & 700 & 100 \\
\hline
\end{tabular}

parameters of the concerned entity. Length of these vectors denotes the probability that a spatial entity exists (Hinton et al. 2011). In CNN, existence of pooling layers can be attributed for most deficiencies but this is not the case with capsule networks since capsule networks uses routing by agreement principle. Using this principle, the generated outputs of the first layer are communicated to parental level capsules of next layer (Sara et al. 2017).

Even though the capsules' coupling coefficient are different, all the capsules attempts to recognize the parental capsules' outputs. Expected conformations to the objectified outputs of the parent capsules causes an increase in the coupling-coefficient of the related capsules (Shahroudnejad et al. 2018). Therefore if output of the capsule $\mathrm{x}$ is $l_{x}$, it's exposure for the parent capsule $y$ is calculated using the formula

$l_{y \mid x}^{\prime}=O_{x y} l_{x}$

where $l_{y \mid x}$ denotes the higher layer yth capsule's expected vector of its output as calculated by the capsule $\mathrm{x}$ in the succeeding layers. The weighted coefficient matrix as learned in the regressive process is $O_{x y}$.

The amount of conformations of the beneath layer and parent capsules work as the foundation of the coupling coefficient $h_{x y}$ can be deduced as

$h_{x y}=\exp \left(g_{x y}\right) / \sum_{z} \exp \left(g_{x z}\right)$

where $g_{x y}$ denotes the probability of $\log$, for deciding whether capsule $\mathrm{x}$ is connected to capsule $\mathrm{y}$ or not and initialized to zero at the beginning of the process. Thus the parent capsule $q_{y}$ input vector can be defined as $q_{y}=\sum_{x} h_{x y} l_{y \mid x}^{\prime}$

The subsequent as mentioned below is applied to ensure that capsules' outputs never go past 1 . Initial vector can be used to define finial outputs of each capsule according to below equation

$m_{y}=\left\|q_{y}\right\|^{2} q_{y} /\left(1+\left\|q_{y}\right\|^{2}\right)\left\|q_{y}\right\|$

where $q_{y}$ and $m_{y}$ denotes the capsule y's input vector and output respectively. In such a routing process, the log updates' probability is numerous, it means that considering the contracts between $m_{y}$ and $l_{y \mid x}$ exploiting the realities of the two supportive vectors, produces a bigger inner product. Hence, contract $f_{x y}$ for updating the log probability as well as coupling coefficient can be calculated as:

$f_{x y}=m_{y} \cdot l_{y \mid x}^{\prime}$

A function that aims high loss values on capsules having long output instigation parameters when the existence of an entity is not recorded is called loss function and it is calculated by:

$t_{z}=R_{z} \max \left(0, m^{+}-\left\|m_{z}\right\|\right)^{2}+\delta\left(1-R_{z}\right) \max \left(0,\left\|m_{z}\right\|-m^{-}\right)^{2}$

where $R_{z}$ is 0 but it is 1 whenever class k exists. The $\delta, m^{-}$, $m^{+}$parameters should be established when learning procedure begins since these are important parameters. The architecture of capsule network consists of a single layer of convolutional filters and two layers of capsules as discussed in (Mukhometzianov and Carrillo 2018). Exceptionally, it needed three layers of interconnected neurons 
Fig. 4 The proposed neurodegenerative diseasesCaps model summary

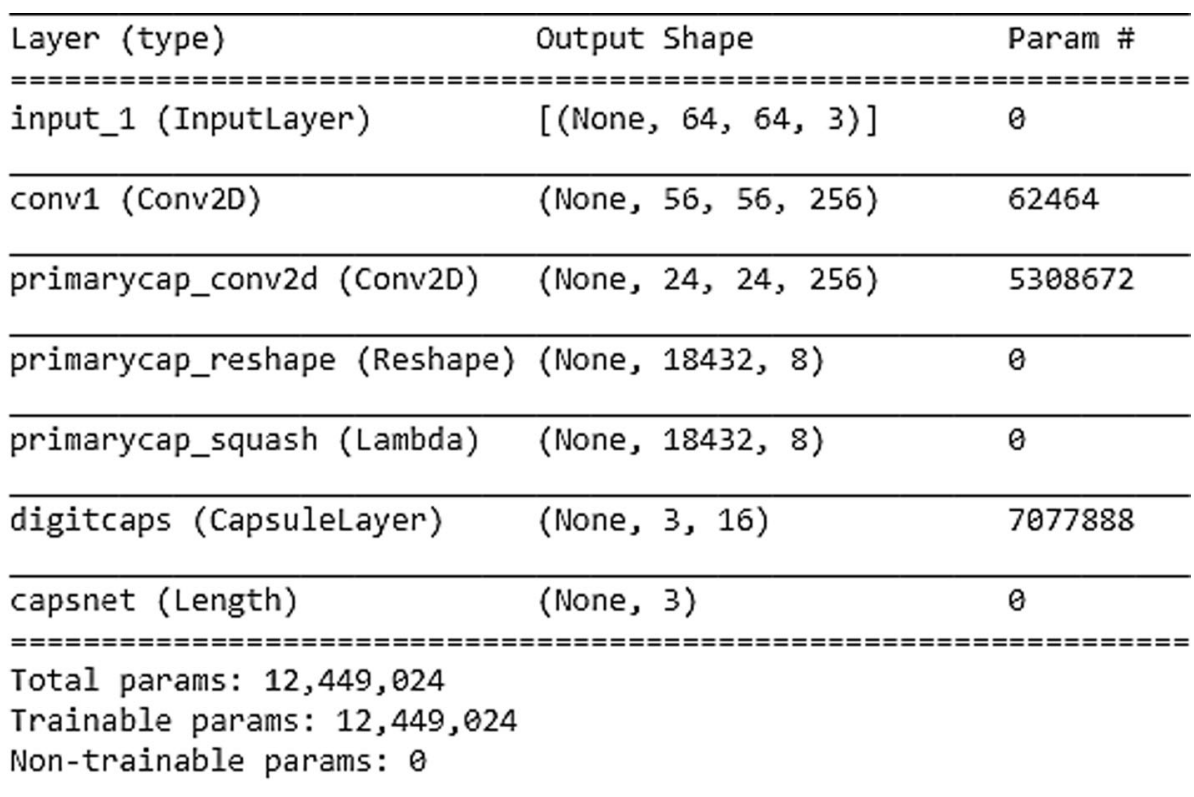

that make an effort to recreate the inputs by means of the instantiation parameters from the capsules associated to true labels.

\section{Designing the neurodegenerative diseases-Caps}

After exploring the numerous architectures of the capsule network, we decided to create a model that consist of four layers along with the Input layer. The layers of our created model is as follows:

1. In order to cut training time as well as for decreasing parameters, the MRI sequences are down-sized from $512 \times 512$ to $480 \times 120$.

2. In our model, $2^{\text {nd }}$ layer is the main capsule layer comprising of 32 channels for convolution where every capsule contains $9 \times 9 \mathrm{kernel}$ and stride value is 2 (Sara et al. 2017).

3. There are 3-capsules in the final year corresponding to 3-class i.e. AD, PD and Healthy control. These two classes actually represents the major Neurodegenerative diseases, we have considered in our study. These capsules has a dimension of 16 .

4. Three connected layers consisting of 512, 1024, 4096 neurons form the decoder segment. To reduce the rebuilding loss neurons in the input layer is equal to the quantity of image pixels in the output layer.

Although considering the risk of over-fitting and underfitting, an early stopping algorithm (Goodfellow et al. 2016) is used but our network achieved actually well on the large size ADPP dataset. Accordingly, the training process will stop when the validation accuracy starts declining at every epoch's end for the period of model training. The model summary of the Neurodegenerative diseases-Caps is presented with the help of Fig. 4 below.

The overall algorithm for this stage is given below as: 


\section{Algorithm 1}

Input: $\mathrm{ADPP}$ dataset consist of sagittal view MRI sequences of $\mathrm{AD}, \mathrm{PD}$ and Healthy controls

Output: The Fine-tuned and trained Capsule Network as the Neurodegenerative diseases-Caps

\section{Steps:}

1. Prepare the ADPP dataset by downloading the sagittal view AD, PD and Healthy control MRI sequences from the ADNI and PPMI databases.

2. Then convert and preprocess these MRI sequences in order to remove the noise, symbols and artifacts.

3. The fine tuning and training of the Capsule network based Neurodegenerative diseases-Caps system. As the capsule network is based on the routing by agreement principle and its step by step procedure as under:

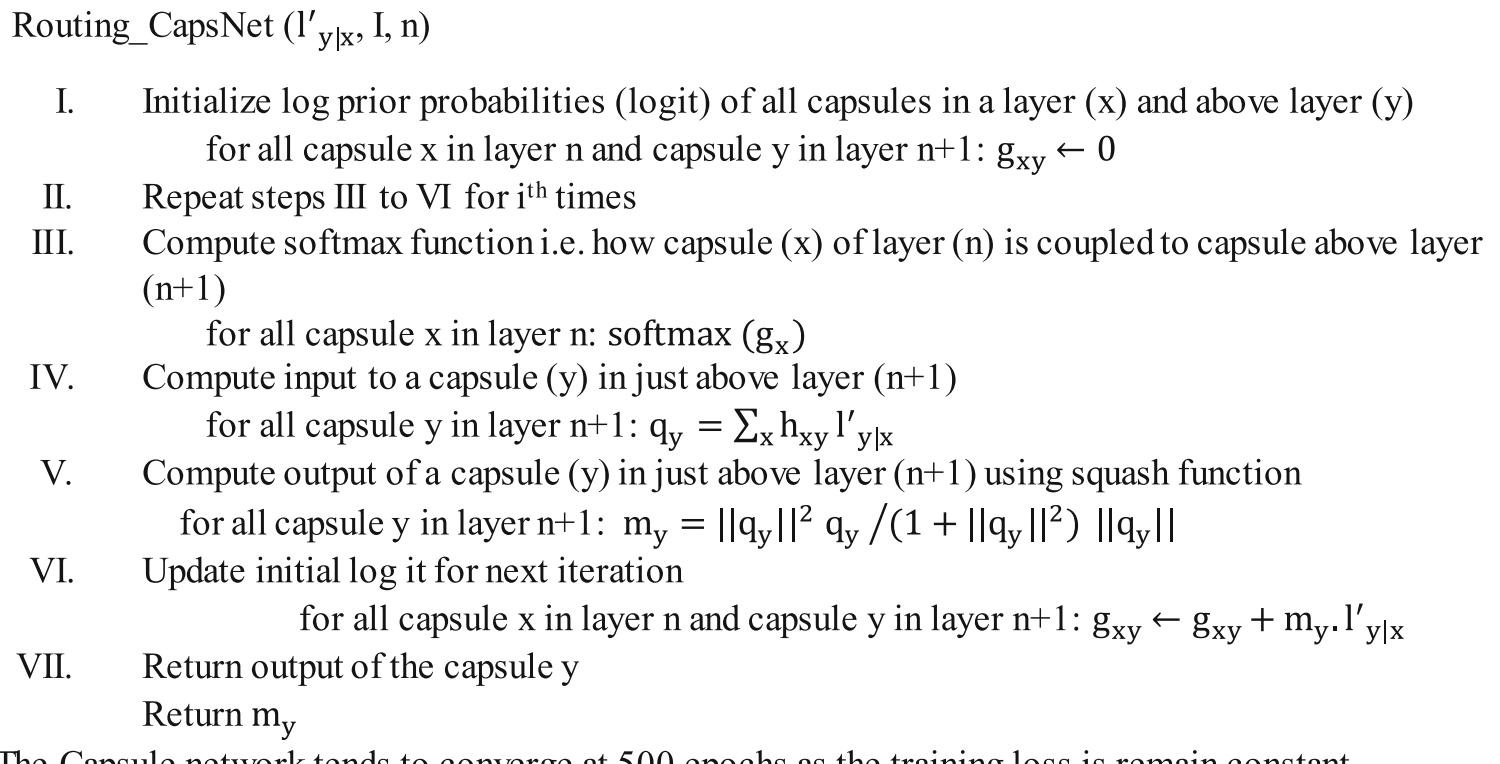

4. The Capsule network tends to converge at 500 epochs as the training loss is remain constant.

\section{Comparison approach}

The four popular deep transfer learning models like VGG16 (Simonyan and Zisserman 2015), VGG19 (Liu and Wang 2019), ResNet50 (He et al. 2016) and InceptionV3 (Szegedy et al. 2014) are used for the sake of comparison and evaluation on the ADPP dataset. These four DTL models are initially fine-tuned and trained using the augmented ADPP dataset. The ultimate object of this comparison is to illustrate that how these commonly used DTL models performs on the ADPP dataset and their performance can be compared with the Capsule network based Neurodegenerative diseases-Caps model. The Algorithm 2 for the comparison approach is given as: 


\section{Algorithm 2}

Input: ADPP dataset consist of sagittal view MRI sequences of AD, PD and Healthy controls

Output: The trained VGG16, VGG 19, ResNet50 and InceptionV3 models for the detection of Neurodegenerative diseases like $\mathrm{AD}$ and $\mathrm{PD}$

\section{Steps:}

1. Preprocess the ADPP dataset in order to remove the noise, symbols and artifacts.

2. Resize the sagittal view MRI sequences to the sizes of 224-by-224-by 3 or 299-by-299-by 3 for the training of these VGG16, VGG 19, ResNet50 and InceptionV3 DTL models.

3. The fine tuning and training of these four DTL models over the augmented datasets.

4. The VGG 16 and VGG19 models tends to converge at 100 epochs.

5. The ResNet 50 and Inception tends to converge at 200 epochs.

6. Simulation and evaluation of these DTL models over the ADPP dataset.

All the four deep transfer learning models are trained and evaluated with a learning rate equal to 0.00001 , minibatch size of 16 and Adam (Kingma 2015) is opted as an optimizer technique for the weights adjustment. Whereas the size of input image and number of epochs required to converge varies from model to model. The dropout method (Srivastava et al. 2014) is utilized in order to avoid the problem of over fitting. The Table 4 below illustrates the configuration parameters of VGG16, VGG19, InceptionV3 and ResNet50 DTL models.

\section{Results and simulation}

The Google Colaboratory (colab) platform powered by the NVidia Tesla T4 GPU along with Python 3.6 is used for the implementation and experimentation. The ADPP dataset is used in the $70 / 30$ split, which means $70 \%$ of ADPP dataset is used for training and remaining $30 \%$ for validation. For testing additional $200 \mathrm{AD}, \mathrm{PD}$ and normal cases MRI sequence are taken as the ADPP testing dataset. The Multiclass confusion matrix of the proposed Capsule network based system over the ADPP testing dataset is presented with the help of Table 5 below. Whereas the Multiclass confusion matrices of the four deep transfer
Table 5 Multi class confusion matrix of the proposed Neurodegenerative diseases-Caps system over the ADPP test dataset for the multiclass i.e. three class classification

\begin{tabular}{llll}
\hline 200/200/200 & Alzeihmer & Parkinson & Healthy control \\
\hline Alzeihmer & $\mathbf{1 9 3}$ & 4 & 3 \\
Parkinson & 1 & $\mathbf{1 9 8}$ & 1 \\
Healthy control & 7 & 8 & $\mathbf{1 8 7}$ \\
\hline
\end{tabular}

learning models over the ADPP testing dataset used for the comparison are illustrated with the help of Table 6 below. The numbers highlighted in bold in the table 5 and 6 are the correctly classified MRI sequences of $\mathrm{AD}, \mathrm{PD}$ and normal cases in the ADPP test dataset by the proposed Neurodegenerative diseases-Caps system and four deep transfer learning based systems used for comparison. From these Multiclass confusion matrices, the values of True positive (TP), True Negative (TN), False Positive (FP) and False Negative (FN) are derived for the Alzheimer class, Parkinson class and Normal or Healthy class. The step by step procedure for determining the values of these TP, TN, FP and FN parameters for each class is somewhat different in Multiclass i.e. three class classification as compare to the conventional binary class classification. Although this step by step procedure for
Table 4 The configuration parameters of VGG16, VGG19, InceptionV3 and ResNet50 DTL models

\begin{tabular}{lllll}
\hline DTL models parameters & VGG16 & VGG19 & InceptionV3 & ResNet50 \\
\hline Input image size & $224 * 224$ & $224 * 224$ & $299 * 299$ & $224 * 224$ \\
Number of layers & 16 & 19 & 48 & 50 \\
Learning rate & 0.00001 & 0.00001 & 0.00001 & 0.00001 \\
Batch size & 16 & 16 & 16 & 16 \\
Number of epochs to converge & 100 & 100 & 200 & 200 \\
Momentum & 0.9 & 0.9 & 0.9 & 0.9 \\
Optimizer & Adam & Adam & Adam & Adam \\
\hline
\end{tabular}


Table 6 Confusion matrices of the (a) VGG 16, (b) VGG19, (c) ResNet50 and (d) Inceptionv3 based systems used for comparison over the ADPP test dataset

\begin{tabular}{llll}
\hline 200/200/200 & Alzeihmer & Parkinson & Healthy control \\
\hline (a) & & & \\
Alzeihmer & $\mathbf{1 7 1}$ & 15 & 14 \\
Parkinson & 10 & $\mathbf{1 7 8}$ & 12 \\
Healthy control & 10 & 18 & $\mathbf{1 7 2}$ \\
(b) & & & \\
Alzeihmer & $\mathbf{1 8 5}$ & 8 & 7 \\
Parkinson & 6 & $\mathbf{1 8 9}$ & 5 \\
Healthy Control & 12 & 14 & $\mathbf{1 7 4}$ \\
(c) & & & \\
Alzeihmer & $\mathbf{1 6 8}$ & 14 & 18 \\
Parkinson & 15 & $\mathbf{1 7 1}$ & 14 \\
Healthy control & 14 & 16 & $\mathbf{1 7 0}$ \\
(d) & & & \\
Alzeihmer & $\mathbf{1 6 4}$ & 16 & 20 \\
Parkinson & 16 & $\mathbf{1 6 8}$ & 16 \\
Healthy control & 18 & 17 & $\mathbf{1 6 5}$ \\
\hline
\end{tabular}

determining the TP, TN, FP and FN values for the Alzheimer class, Parkinson class and Normal class is clearly illustrated with the help of Appendix 1. These derived TP, TN, FP and FN values for each class i.e. Alzheimer, Parkinson and Healthy control are finally used in the formulas of various statistical parameters or classification rates in order to determine the performance evaluation of this proposed system. The derived TP, TN, FP and FN values for the Alzheimer class, Parkinson class and Healthy control class of the proposed Neurodegenerative diseasesCaps system over the ADPP testing dataset are presented with the help of Table 7 .

The statistical parameters or classification rates like Accuracy, Sensitivity, Specificity and F1-score etc., which are used for the performance evaluation are well defined in the Table 8 below. The Tables 8,9 and 10 below simply illustrates the performance of the proposed system as well as the VGG16, VGG19, InceptionV3 and ResNet50 DTL models over the ADPP dataset for the multiclass classification of Neurodegenerative diseases. The Fig. 5 below simply illustrates the ROC curve, training loss and training $\&$ validation accuracy graphs of the proposed Neurodegenerative-Caps system. The Fig. 6 below simply showcase the performance comparison graph for comparing the performance of Capsule network based proposed system with the VGG19, VGG16, ResNet50 and InceptionV3 based system.

Whereas the training and validation graph of four DTL models on ADPP dataset is presented with the help of Fig. 7 above. The training loss curve of all the four DTL models used for the comparison are presented with the help of Fig. 8 above. The proposed Capsule network based system is also compared with some of the state of the art
Table 7 The TP, TN, FP and FN values obtained from the Multiclass confusion matrix of the proposed Neurodegenerative diseases-Caps system

\begin{tabular}{llll}
\hline Values/class & Alzheimer class & Parkinson class & Healthy control class \\
\hline True positive & 193 & 198 & 185 \\
True negative & 392 & 389 & 396 \\
False positive & 7 & 2 & 15 \\
False negative & 8 & 12 & 4 \\
\hline
\end{tabular}

Table 8 Performance of the proposed Neurodegenerative diseases-Caps system on the ADPP dataset

\begin{tabular}{lllll}
\hline Classification rates & Their formulas & \multicolumn{2}{c}{ Multiclass classification into } \\
\cline { 3 - 5 } & & Alzheimer & Parkinson & Healthy control \\
\hline Accuracy & $(\mathrm{TP}+\mathrm{TN}) /(\mathrm{TP}+\mathrm{TN}+\mathrm{FP}+\mathrm{FN})$ & 97.81 & 98 & 96.81 \\
Sensitivity & $\mathrm{TP} /(\mathrm{TP}+\mathrm{FN})$ & 96.02 & 94.2 & 97.8 \\
Specificity & $\mathrm{TN} /(\mathrm{FP}+\mathrm{TN})$ & 98.25 & 99 & 96.66 \\
Precision & $\mathrm{TP} /(\mathrm{TP}+\mathrm{FP})$ & 96.5 & 99 & 92.5 \\
Negative predictive value & $\mathrm{TN} /(\mathrm{TN}+\mathrm{FN})$ & 97.89 & 97 & 0.005 \\
False positive rate & $\mathrm{FP} /(\mathrm{FP}+\mathrm{TN})$ & 0.0175 & 0.01 & 0.0332 \\
False discovery rate & $\mathrm{FP} /(\mathrm{FP}+\mathrm{TP})$ & 0.035 & 0.57 & 0.0750 \\
False negative rate & $\mathrm{FN} /(\mathrm{FN}+\mathrm{TP})$ & 2.03 & 96.24
\end{tabular}

$T P$ True positive, $T N$ true negative, $F P$ false positive, $F N$ false negative 
Table 9 The performance of the VGG 19 and VGG16 based systems on the ADPP dataset

\begin{tabular}{|c|c|c|c|c|c|c|}
\hline \multirow[t]{3}{*}{ Classification rates } & \multicolumn{6}{|c|}{ Multiclass classifications into } \\
\hline & \multicolumn{2}{|c|}{ Alzheimer } & \multicolumn{2}{|c|}{ Parkinson } & \multicolumn{2}{|c|}{ Healthy control } \\
\hline & VGG19 & VGG16 & VGG19 & VGG16 & VGG19 & VGG16 \\
\hline Accuracy & 94.6 & 92 & 95 & 91 & 94 & 91 \\
\hline Sensitivity & 91.13 & 90 & 90 & 85 & 94 & 87 \\
\hline Specificity & 96.22 & 92.9 & 97 & 94 & 94 & 93.03 \\
\hline Precision & 92.5 & 86 & 95 & 89 & 87 & 86 \\
\hline Negative predictive value & 95.5 & 95 & 95.5 & 92 & 97 & 93.5 \\
\hline False positive rate & 0.037 & 0.070 & 0.028 & 0.056 & 0.0628 & 0.069 \\
\hline False discovery rate & 0.075 & 0.145 & 0.055 & 0.11 & 0.13 & 0.14 \\
\hline False negative rate & 0.088 & 0.10 & 0.104 & 0.156 & 0.064 & 0.13 \\
\hline F1 score & 91.8 & 87.4 & 92 & 86.6 & 90.16 & 86.4 \\
\hline
\end{tabular}

Table 10 The performance of ResNet50 and InceptionV3 based systems on the ADPP dataset

\begin{tabular}{|c|c|c|c|c|c|c|}
\hline \multirow[t]{3}{*}{ Classification rates } & \multicolumn{6}{|c|}{ Multiclass classifications into } \\
\hline & \multicolumn{2}{|l|}{ Alzheimer } & \multicolumn{2}{|l|}{ Parkinson } & \multicolumn{2}{|c|}{ Healthy control } \\
\hline & ResNet50 & InceptionV3 & ResNet50 & InceptionV3 & ResNet50 & InceptionV3 \\
\hline Accuracy & 89.8 & 88 & 90.17 & 89 & 89.6 & 88 \\
\hline Sensitivity & 85.2 & 83 & 85.07 & 83.5 & 84.14 & 82.09 \\
\hline Specificity & 92 & 91 & 92 & 91.9 & 92.46 & 91.2 \\
\hline Precision & 84 & 82 & 85.5 & 84 & 85 & 82.5 \\
\hline Negative predictive value & 92 & 91.5 & 92.5 & 91.7 & 91.89 & 91 \\
\hline False positive rate & 0.079 & 0.089 & 0.072 & 0.0802 & 0.075 & 0.087 \\
\hline False discovery rate & 0.16 & 0.18 & 0.145 & 0.16 & 0.15 & 0.175 \\
\hline False negative rate & 0.147 & 0.1717 & 0.149 & 0.16 & 0.158 & 0.179 \\
\hline F1 score & 84.6 & 82.4 & 85.2 & 83.7 & 84.58 & 82.2 \\
\hline
\end{tabular}

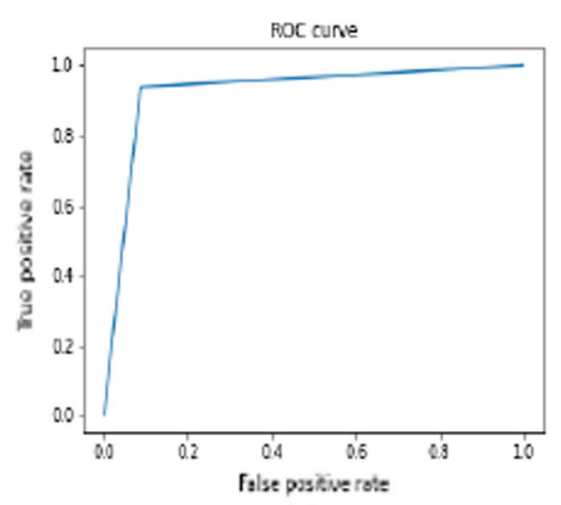

(a)

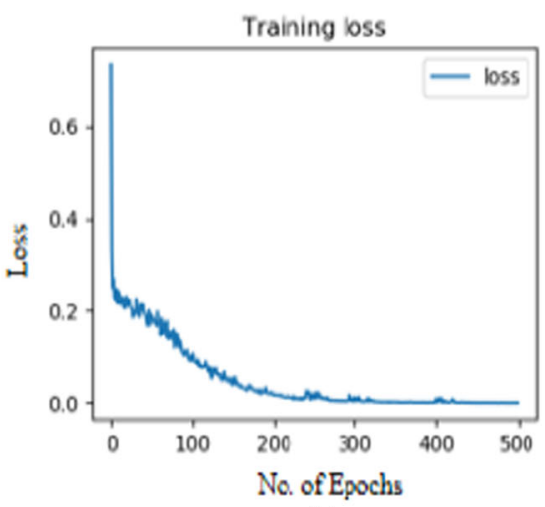

(b)

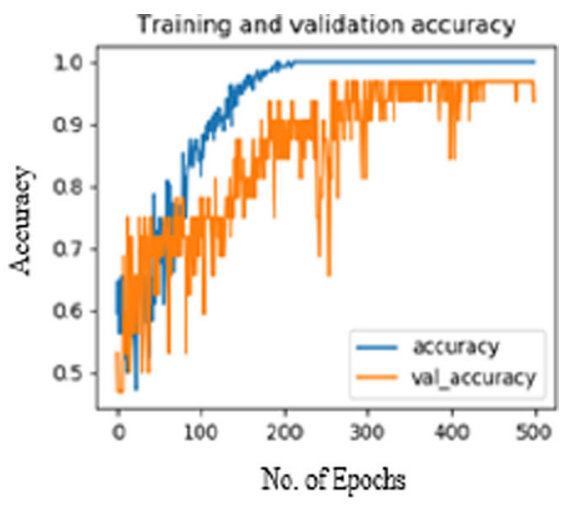

(c)

Fig. 5 The ROC curve (a), training loss curve (b) and training and validation accuracy graph (c) of the proposed Neurodegenerative-Caps system

approaches proposed in the recent years for either performing the Alzheimer classification or Parkinson classification on the ADNI or PPMI datasets. Their comparison is presented with the help of Tables 11 and 12. The classification rates of the proposed Neurodegenerative diseases-Caps system on the ADNI and PPMI datasets are highlighted in bold in table 11 and 12 . 
Fig. 6 The performance comparison graph for comparing the performance of Capsule network based proposed system with the VGG19, VGG16, ResNet50 and InceptionV3 based system

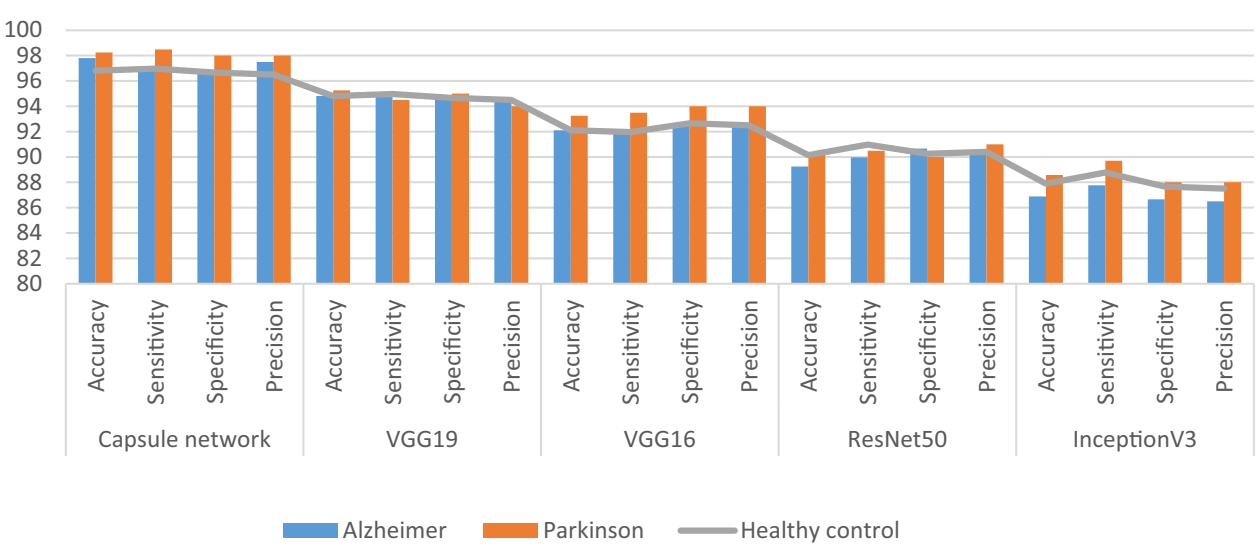

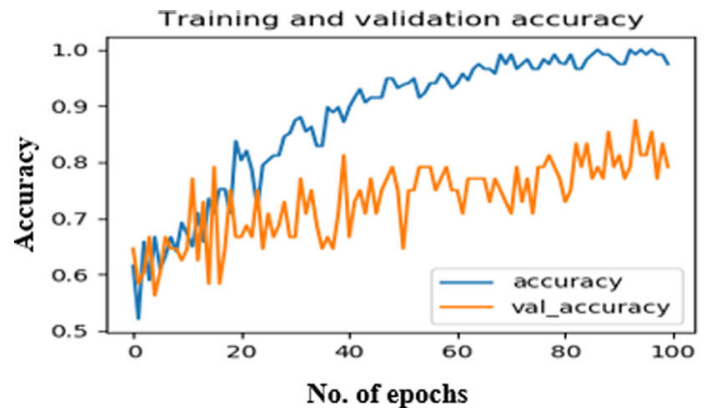

(a)

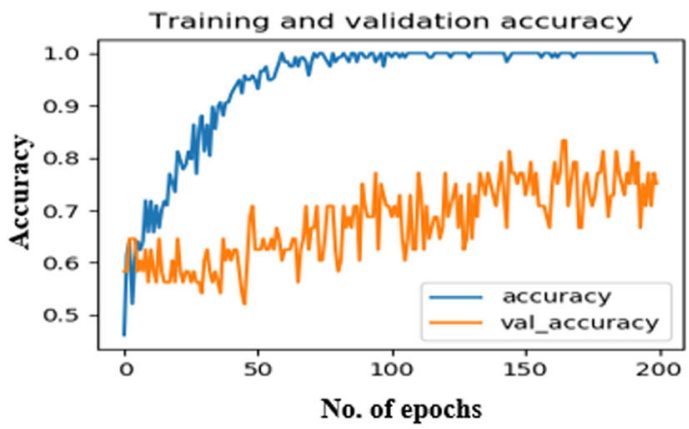

(c)

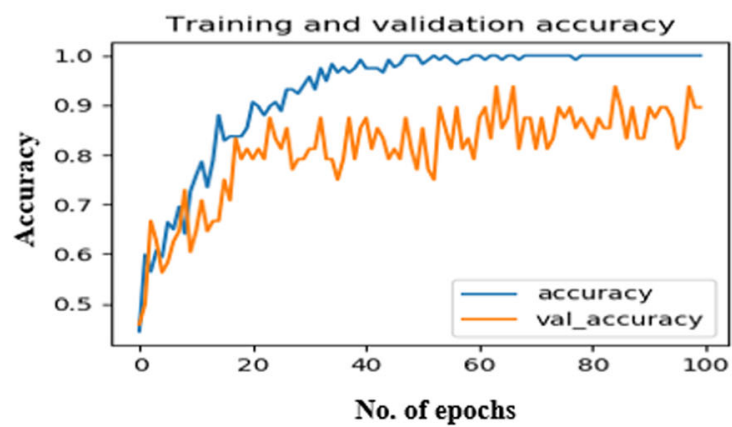

(b)

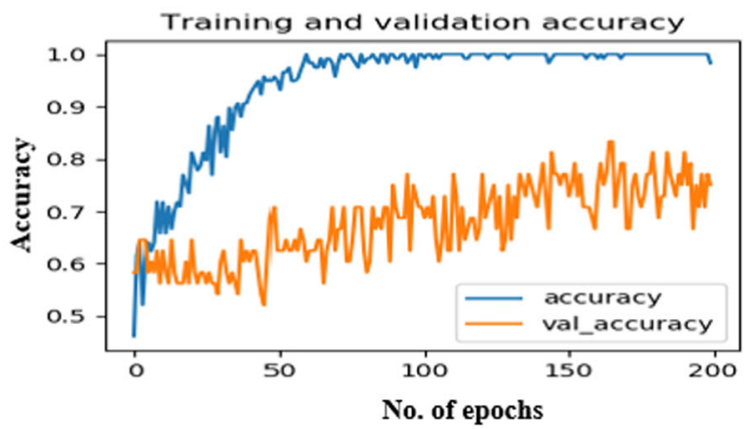

(d)

Fig. 7 The training and validation graph of four DTL models on ADPP dataset a VGG16, b VGG19, c ResNet50, d InceptionV3

\section{Discussion}

The proposed Neurodegenerative disease-Caps system based on the Capsule network is proved to be very accurate especially in terms of performing the multiclass classification as compare to other deep transfer learning models. The major reason of employing the Capsule network is its ability to deal with affine transformations and invariance (rotational and translational), which very commonly persists in the MRI modalities datasets. This type of automated systems can be very effectively used for doing the early screening of patients suffering from the Neurodegenerative disease. The need of such systems is even more felt or realized in the developing and densely populated countries, where patient's to doctor ratio is drastically low. Such automated system can enhance the diagnostic capabilities as well as efficiency of the radiologists. As the radiologists are more inclined towards using the sagittal view MRI sequence for the correct diagnosis of these two Neurodegenerative diseases as compare to the axial and coronal view. So we have decided to utilize this sagittal view MRI sequence for performing the experimentation in this research study. Another important reason of utilizing this view of MRI sequence only is the availability of high quality MRI sequences, which are selected with the aid of radiologist and tends to deliver better results. There are already approaches based on machine and deep learning utilizing the axial view of MRI sequence for performing 

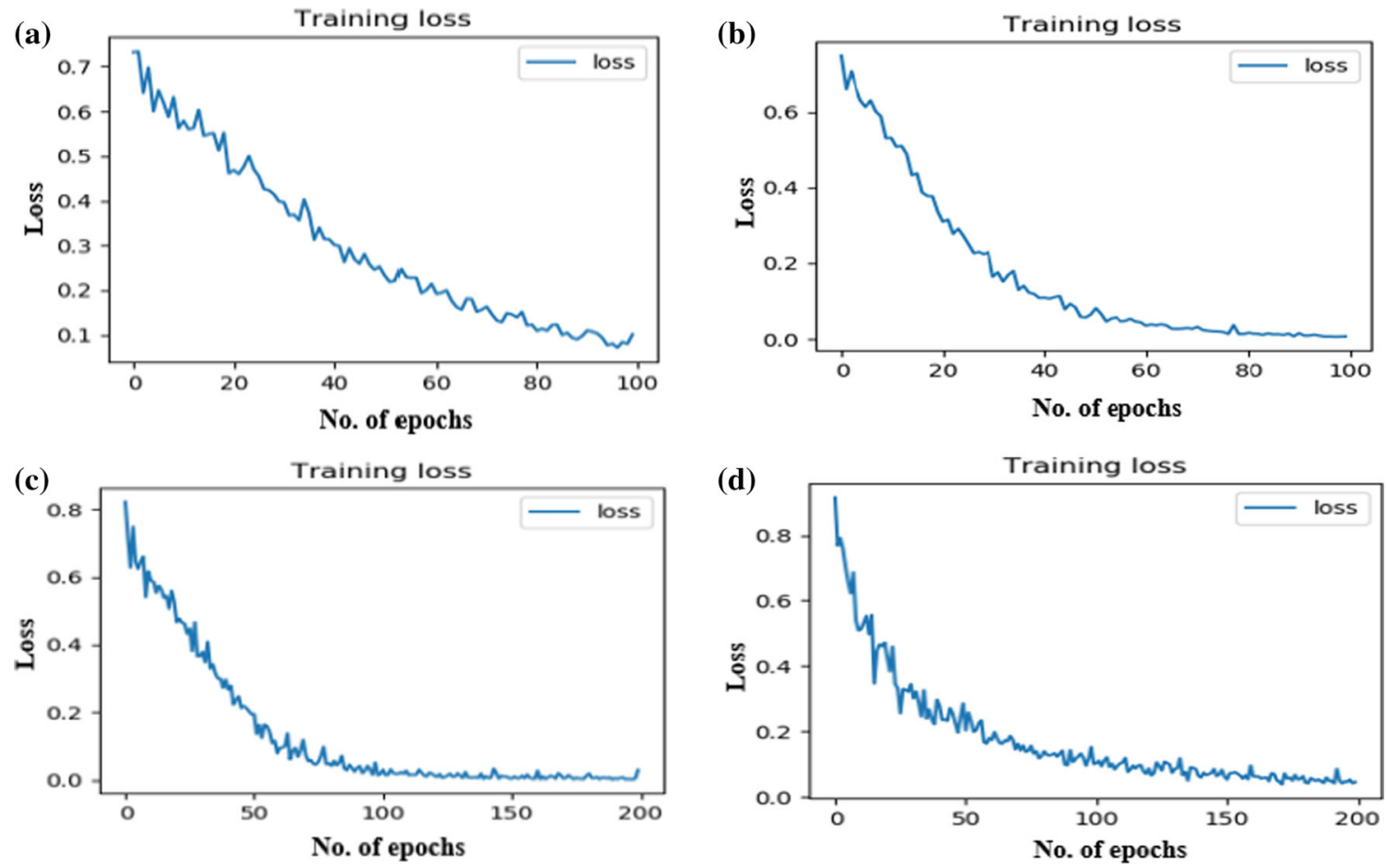

Fig. 8 The training loss graph of four DTL models on ADPP dataset a VGG16, b VGG19, c ResNet50, d InceptionV3

Table 11 Performance comparison of the proposed Neurodegenerative disease-Caps system with the existing approaches for the Alzheimer classification on the ADNI dataset

\begin{tabular}{|c|c|c|c|c|c|}
\hline Author and year & Machine learning classifier or deep learning model used & $\begin{array}{l}\text { Accuracy } \\
(\%)\end{array}$ & $\begin{array}{l}\text { Precision } \\
(\%)\end{array}$ & $\begin{array}{l}\text { Sensitivity } \\
(\%)\end{array}$ & F1 score $(\%)$ \\
\hline Li et al. $(2014 a, b)$ & Support vector machine & 83 & 82.8 & 80.4 & 81 \\
\hline Glozman et al. (2017) & Support vector machine & 80.54 & 81 & 70.59 & 76 \\
\hline Talo et al. 2019 & ResNet34 & 81.3 & 80.5 & 79.9 & 79 \\
\hline Proposed one & Capsule network & 97.81 & 96.5 & 96.02 & 96.26 \\
\hline
\end{tabular}

Table 12 Performance comparison of the proposed Neurodegenerative disease-Caps system with the existing approaches for the Parkinson's disease classification on the PPMI dataset

\begin{tabular}{|c|c|c|c|c|c|}
\hline Author and year & $\begin{array}{l}\text { Machine learning classifier or deep learning model } \\
\text { used }\end{array}$ & $\begin{array}{l}\text { Accuracy } \\
(\%)\end{array}$ & $\begin{array}{l}\text { Precision } \\
(\%)\end{array}$ & $\begin{array}{l}\text { Sensitivity } \\
(\%)\end{array}$ & $\begin{array}{l}\text { F1 score } \\
(\%)\end{array}$ \\
\hline Peng et al. (2017) & Multi-kernel support vector machine (SVM) & 85.78 & 85 & 87.64 & 86.2 \\
\hline $\begin{array}{l}\text { Sivaranjini et al. } \\
\text { (2019) }\end{array}$ & Alexnet & 88.9 & 87.5 & 89.3 & 88.2 \\
\hline Yagis et al. (2019) & VGG16 and ResNet 50 & 82 & 81.2 & 80.1 & 81.9 \\
\hline Proposed one & Capsule network & 98 & 99 & 94.2 & 96.24 \\
\hline
\end{tabular}

the classification of either Alzheimer into sub stages or Parkinson but the experimentation is still required to be done with the sagittal view. The sagittal view MRI sequence of human brain offers more information related to the specific Alzheimer as well Parkinson affected brain regions like rostral hippocampus, rostral hippocampus, Medial amygdala, Medial amygdala, caudal area etc. in 2D as compare to the axial and coronal view. The sagittal view 
MRI sequence thus proved to be more efficient and reliable for the correct classification of these Neurodegenerative diseases and this fact is very proved in the result section.

An experimentation that involves feeding of the proposed Neurodegenerative diseases-Caps system with input MRI sequences with different sizes are done. This experimentation is done in order to reduce the training time quotient of the proposed model. The different size MRI sequences are tried as input in order to find the best input size that offers the least training time while offering the best accuracy quotient. As the proposed Capsule network based model is offering the highest training time with input as $512 * 512$ size MRI sequence. Whereas the proposed model offered the best accuracy quotient with least training time with input of $480 * 120$ size MRI sequence, so this input size is used in the research study. The other input sizes that are used in the experimentation are $512 * 480$, $480 * 480,480 * 240,480 * 60$ etc. This experimentation with different MRI input sizes is also done in order to observe or determine that how the proposed capsule network based model responds to the low resolution input images in the training phase. This experimentation has given answers to questions like:

1. Whether the proposed model can deliver good results with low resolution images?

2. Whether the proposed model is able to better optimize itself whenever trained with low resolution input images etc.?

So, yes the proposed model is delivering best accuracy with least training time with $480 * 120$ size MRI sequence. Another very important observation of this experimentation is that the proposed model is able to better optimize itself during the training phase, whenever trained with input MRI sequence with resolution of $480 * 120$ in order to classify the test images correctly later in the testing phase. The parameters associated with the Capsule network for the proper tuning are decided after performing a number of experiments. The Capsule network tends to converges at 500 epochs during training and after that its validation accuracy is not at all improving.

The performance comparison along with the more complex deep transfer learning models in the result section simply proves that the Capsule network based system is more robust and accurate. Whereas the other four DTL models which tends to converge much earlier during training at either 100 or 200 epochs. Apart from the fact, that the Capsule network are taking more time to converge and requires lot of hardware resources, there are no other drawback, which can be associated with this proposed system. The major reasons behind the better performance of the proposed Neurodegenerative disease-Caps system in comparison to the deep transfer learning models over the ADPP dataset are as follows:

1. The proposed Neurodegenerative disease-Caps system is based on the routing by agreement principle, so it is generalizing the invariance and affine transformation infected input test samples correctly. Whereas the four deep transfer learning models are completely fail to generalize such test samples as these DTL models are based on the pooling layers concept.

2. The deep transfer learning models performs well on the very large size training datasets, which is not the case here as the size of the ADPP dataset is not very large.

3. The Capsule networks allows the experimentation to be done with the input size of training samples. This type of experimentation will helps in identifying the correct input size samples, which proved to be accurate and training time efficient. Whereas the four deep transfer learning models are rigid in terms of size of input samples and provides no scope for experimentation.

4. The proposed Capsule network based system is far less complex as it consist of small number of layers i.e. (4 convolution layers + primary Input capsule layer). This less complex nature of the proposed system makes the hyper parameter tuning part easy and hence result in more accurate model. Unlike deep transfer learning models, which consist of large number of layers and making the task of hyper parameter tuning a difficult one.

\section{Conclusion}

To the best of our knowledge, there is no system proposed yet for the multiclass classification of major Neurodegenerative diseases like Alzheimer and Parkinson. There are deep learning based automated approaches for the classification of either Alzheimer or Parkinson diseases alone. So the proposed Neurodegenerative disease-Caps system is a unified framework based on Capsule network for the multiclass classification of these Neurodegenerative diseases. This systems delivers an encouraging accuracy of above 97, 98 and $96 \%$ for the correct classification of Alzheimer, Parkinson and Normal cases. Whereas the VGG19, VGG16, ResNet50 and InceptionV3 DTL models delivers average accuracies of 92, 90, 89 and $87 \%$ respectively. The result section moreover proves that the proposed Neurodegenerative disease-Caps system based on Capsule Network outperforms the VGG19, VGG16, ResNet50 and InceptionV3 models in terms of accuracy and performance. In future, quotient of multiclass classification of this system can be further enhanced in order to 
perform the classification of other brain structural disorders like brain tumors, Schizophrenia, dementia etc.

Supplementary Information The online version contains supplementary material available at https://doi.org/10.1007/s11571022-09787-1.

Acknowledgements We like to thank Dr. Pushraj Bhatele (Chief Radiologist) of MP MRI and CT scan center at MP MRI and CT scan center, Netaji Subhash Chandra Bose Medical College, Jabalpur, India for providing his valuable support in terms of medical knowledge and validate this proposed work. We also like to extend our gratitude to the Sanya Hospital and Diagnostics Pvt. Ltd. Delhi for supporting this research study.

Funding This research study is supported by the Sanya Hospital and Diagnostics Pvt. Ltd. Delhi.

Availability of data and materials The dataset used in this research is publically available.

Code availability The code will be provided on request.

\section{Declarations}

Conflict of interest The authors declare that they have no conflict of interest.

Ethical approval This article does not contain any studies with human participants or animals performed by any of the authors.

Consent to participate This article does not contain any studies with human participants or animals performed by any of the authors.

Consent for publication All the authors have given their consent.

\section{References}

Adeli E, Shi F, An L, Wee CY, Wu G, Wang T et al (2016) Joint feature-sample selection and robust diagnosis of Parkinson's disease from MRI data. J Neuroimaging 141:206-219. https:// doi.org/10.1016/j.neuroimage.2016.05.054

Adu K, Yu Y, Cai J, Owusu-Agyemang K, Twumasi BA, Wang X (2021a) DHS-CapsNet: dual horizontal squash capsule networks for lung and colon cancer classification from whole slide histopathological images. Int $\mathrm{J}$ Imaging Syst Technol 31(4):2075-2092. https://doi.org/10.1002/ima.22569

Adu K, Yu Y, Cai J, Mensah PK, Owusu-Agyemang K (2021b) MLAF-CapsNet: multi-lane atrous feature fusion capsule network with contrast limited adaptive histogram equalization for brain tumor classification from MRI images. J Intell Fuzzy Syst 41(2):3933-3950

Afshar P, Heidarian S, Naderkhani F, Oikonomou A, Plataniotis KN, Mohammadi A (2020) COVID-CAPS: a capsule network-based framework for identification of COVID-19 cases from X-ray images. Pattern Recogn Lett 138:638-643. https://doi.org/10. 1016/j.patrec.2020.09.010

Ali M, Ali R (2021) Multi-input dual-stream capsule network for improved lung and colon cancer classification. Diagnostics 11(8):1485. https://doi.org/10.3390/diagnostics11081485

Association A (2017) Alzheimer's disease facts and figures. Alzheimer's Dementia 13(4):325-373 (Accessed 25th July 2021)
Amoroso N, La Rocca M, Monaco A, Bellotti R, Tangaro S (2018) Complex networks reveal early MRI markers of Parkinson's disease. Med Image Anal 48:12-24. https://doi.org/10.1016/j. media.2018.05.004

Bakator M, Radosav D (2018) Deep learning and medical diagnosis: a review of literature. Multimodal Technol Interact 2(3):47. https://doi.org/10.3390/mti2030047

Borek LL, Amick MM, Friedman JH (2006) Non-motor aspects of Parkinson's disease. CNS Spectrum 11(7):541-554. https://doi. org/10.1017/s1092852900013560

Chakraborty S, Aich S, Kim HC (2020) Detection of Parkinson's disease from 3T T1 Weighted MRI scans using 3D convolutional neural network. Diagnostics 10(6):402-419. https://doi.org/10. 3390/diagnostics10060402

Choi H, Jin KH (2018) Alzheimer's disease neuroimaging predicting cognitive decline with deep learning of brain metabolism and amyloid imaging. Behavior Brain Resonance 344:103-109

Doan NT, Engvig A, Zaske K, Persson K, Lund MJ, Kaufmann T et al (2017) Distinguishing early and late brain aging from the Alzheimer's disease spectrum: consistent morphological patterns across independent samples. Neuroimaging 158:282-295. https://doi.org/10.1016/j.neuroimage.2017.06.070

Dyrba M, Grothe M, Kirste T, Teipel SJ (2015) Multimodal analysis of functional and structural disconnection in Alzheimer's disease using multiple kernels SVM. Hum Brain Mapp 36(6):2118-2131. https://doi.org/10.1002/hbm.22759

El-Sappagh S, Abuhmed T, Riazul Islam SM, Kwak KS (2020) Multimodal multitask deep learning model for Alzheimer's disease progression detection based on time series data. Neurocomputing 418:197-215

Farzan A, Mashohor S, Ramli AR, Mahmud R (2015) Boosting diagnosis accuracy of Alzheimer's disease using high dimensional recognition of longitudinal brain atrophy patterns. Behav Brain Res 290:124-130. https://doi.org/10.1016/j.bbr.2015.04. 010

Feng W, Halm-Lutterodt NV, Tang H, Mecum A, Mesregah MK, Ma Y, Guo X (2020) Automated MRI-based deep learning model for detection of Alzheimer's disease process. Int J Neural Syst 30(6):2050032. https://doi.org/10.1142/S012906572050032X

Ferreri F, Agbokou C, Gauthier S (2006) Recognition and management of neuropsychiatric complications in Parkinson's disease. J L'assoc Med Can 175(12):1545-1552. https://doi.org/10.1503/ cmaj.060542

Fulton LV, Dolezel D, Harrop J, Yan Y, Fulton CP (2019) Classification of Alzheimer's disease with and without Imagery using gradient boosted machines and ResNet-50. Brain Sci 9(9):212. https://doi.org/10.3390/brainsci9090212

Garali I, Adel M, Bourennane S, Guedj E (2018) Histogram-based features selection and volume of interest ranking for brain PET image classification. IEEE J Transl Eng Health Med 6:2100212. https://doi.org/10.1109/JTEHM.2018.2796600

Goodfellow IJ, Bengio Y, Courville A (2016) Deep learning. Cambridge MIT Press. https://doi.org/10.4258/hir.2016.22.4.351

Glozman T, Solomon J, Pestilli F, Guibas L (2017) Alzheimer's disease neuroimaging: shape-attributes of brain structures as biomarkers for Alzheimer's disease. J Alzheimer's Dis 56(1):287-295. https://doi.org/10.3233/JAD-160900

Goceri E (2019) Diagnosis of Alzheimer's disease with Sobolev gradient-based optimization and 3D convolutional neural network. Int J Numer Methods Biomed Eng 35:e3225. https://doi. org/10.1002/cnm.3225

Guo H, Zhang F, Chen J, Xu Y, Xiang J (2017) Machine learning classification combining multiple features of a hyper-network of fMRI data in Alzheimer's disease. Front Neurosci 11:615. https://doi.org/10.3389/fnins.2017.00615 
He K, Zhang X, Ren S, Sun J (2016) Deep residual learning for image recognition. CVPR, pp 770-778. arXiv:1512.03385

Heidarian S, Afshar P, Enshaei N et al (2021) COVID-FACT: a fullyautomated capsule network-based framework for identification of COVID-19 cases from chest CT scans. Front Artif Intell 4:598932. https://doi.org/10.3389/frai.2021.598932

Hinton GE, Krizhevsky A, Wang SD (2011) Transforming autoencoders. In: Lecture notes in computer science. Springer, Berlin, Heidelberg, pp 44-51. https://doi.org/10.1007/978-3642-21735-7_6

Jiménez-Sánchez A, Albarqouni S, Mateus D (2018) Capsule networks against medical imaging data challenges. In: Intravascular imaging and computer assisted stenting and large-scale annotation of biomedical data and expert label synthesis. Springer, Cham, pp 150-60. https://doi.org/10.1007/978-3-03001364-6 17

Kingma DP (2015) ADAM: a method for stochastic optimization. arXiv:1412.6980

Li M, Qin Y, Gao F, Zhu W, He X (2014a) Discriminative analysis of multivariate features from structural MRI and diffusion tensor images. Magn Reson Imaging 32(8):1043-1051. https://doi.org/ 10.1016/j.mri.2014.05.008

Li M, Oishi K, He X, Qin Y, Gao F, Mori S et al (2014b) An efficient approach for differentiating Alzheimer's disease from normal elderly based on multicentre MRI using gray-level invariant features. J PLoS One 9(8):e105563. https://doi.org/10.1371/ journal.pone. 0105563

Liu T, Wang Z (2019) HiCNN: a very deep convolutional neural network to better enhance the resolution of Hi-C data. Bioinformatics 35(21):4222-4228. https://doi.org/10.1093/bioinfor matics/btz251

Lundervold AS, Lundervold A (2019) An overview of deep learning in medical imaging focusing on MRI. Z Med Phys 29:102-127. https://doi.org/10.1016/j.zemedi.2018.11.002

Marek K, Jennings D, Lasch S, Siderowf A, Tanner C, Simuni T, Coffey C, Kieburtz K et al (2011) The Parkinson progression marker initiative (PPMI). Prog Neurobiol 95:629-635

McKhann G, Drachman D, Folstein M, Katzman R, Price D, Stadlan EM (1984) Clinical diagnosis of Alzheimer's disease: Report of the nincds-adrda work group* under the auspices of department of health and human services task force on Alzheimer's disease. Neurology 34(7):939-944. https://doi.org/10.1212/wnl.34.7.939

Mozhdehfarahbakhsh A, Chitsazian S, Chakrabarti P, Chakrabarti T, Kateb B, Nami M (2021) An MRI-based deep learning model to predict Parkinson's disease stages. medRxiv. https://doi.org/10. 1101/2021.02.19.21252081

Mukhometzianov R, Carrillo J (2018) CapsNet comparative performance evaluation for image classification. arXiv:1805.11195

Naz S, Ashraf A, Zaib A (2021) Transfer learning using freeze features for Alzheimer neurological disorder detection using ADNI dataset. Multimedia Syst. https://doi.org/10.1007/s00530021-00797-3

Ni H, Zhou L, Ning X, Wang L (2016) Alzheimer's disease neuroimaging: exploring multi fractal-based features for mild Alzheimer's disease classification. Magn Reson Med 76(1):259-269. https://doi.org/10.1002/mrm.25853

Oliveira FPM, Faria DB, Costa DC, Castelo-Branco M, Tavares JMRS (2017) Extraction, selection and comparison of features for an effective automated computer-aided diagnosis of Parkinson's disease based on FP-CIT SPECT images. Eur J Nucl Med Mol Imaging 45(6):1052-1062. https://doi.org/10.1007/s00259017-3918-7

Peng B, Wang S, Zhou Z, Liu Y, Tong B, Zhang T et al (2017) A multilevel-ROI-features-based machine learning method for detection of morph metric biomarkers in Parkinson's disease. Neurosci Lett Elsevier 651:88-94. https://doi.org/10.1016/j. neulet.2017.04.034

Ramzan F, Khan MUG, Rehmat A, Iqbal S, Saba T, Rehman A, Mehmood Z (2020) A deep learning approach for automated diagnosis and multi-class classification of Alzheimer's disease stages using resting-state fMRI and residual neural networks. J Med Syst 44:37. https://doi.org/10.1007/s10916-019-1475-2

Russakovsky O, Deng J, Su H, Krause J, Satheesh S, Ma S, Huang Z, Karpathy A, Khosla A, Bernstein M, Berg AC, Fei-Fei L (2015) ImageNet large scale visual recognition challenge. Int J Comput vis 115(3):211-252. https://doi.org/10.1007/s11263-015-0816-y

Sabour S, Frosst N, Hinton GE (2017) Dynamic routing between capsules. In: 31st conference on neural information processing systems, Long Beach, CA, USA

Sara S, Nicholas F, Hinton GE (2017) Dynamic routing between capsules. In: Proceedings of the 31st international conference on neural information processing systems, pp 3859-3869

Shahroudnejad A, Mohammadi A, Plataniotis KN (2018) Improved explainability of capsule networks: relevance path by agreement. arXiv: $1802.10204 \mathrm{v} 1$

Simonyan K, Zisserman A (2015) Very deep convolutional networks for large-scale image recognition. arXiv:1409.1556

Srivastava N, Hinton G, Krizhevsky A, Sutskever I, Salakhutdinov R (2014) Dropout: a simple way to prevent neural networks from over fitting. J Mach Learn Res 15(56):1929-1958

Szegedy C, Liu W, Jia Y, Sermanet P, Reed S, Anguelov D, Erhan D, Vanhoucke V, Rabinovich A (2014) Going deeper with convolutions. Technical report, pp 1-25. arXiv:1409.4842

Talo M, Baloglu UB, Yıldırım Ö, Acharya R (2019) Application of deep transfer learning for automated brain abnormality classification using MR images. Cogn Syst Res 54:176-188. https:// doi.org/10.1016/j.cogsys.2018.12.007

Tiwari S, Jain A (2021) Convolutional capsule network for COVID19 detection using radiography images. Int J Imaging Syst Technol. https://doi.org/10.1002/ima.22566

Vyas T, Yadav R, Solanki C, Darji R, Desai S, Tanwar S (2021) Deep learning-based scheme to diagnose Parkinson's disease. Expert Syst. https://doi.org/10.1111/exsy.12739

Wang SH, Phillips P, Sui Y, Liu B, Yang M, Cheng H (2018) Classification of Alzheimer's disease based on eight-layer convolutional neural network with leaky rectified linear unit and max pooling. J Med Syst 42(5):85. https://doi.org/10.1007/ s10916-018-0932-7

Wang P, Wang J, Li Y, Li P, Li L, Jiang M (2021) Automatic classification of breast cancer histopathological images based on deep feature fusion and enhanced routing. Biomed Signal Process Control 65:102341. https://doi.org/10.1016/j.bspc.2020. 102341

Worrall DE, Garbin SJ, Turmukhambetov D, Brostow GJ (2017) Harmonic networks: deep translation and rotation equivariance. In: IEEE conference on computer vision and pattern recognition IEEE computer society, pp 7168-7177. https://doi.org/10.1109/ CVPR.2017.758

Yagis E, De Herrera AGS, Citi L (2019) Generalization performance of deep learning models in neurodegenerative disease classification. In: IEEE international conference on bioinformatics and biomedicine (BIBM), San Diego, USA, pp 1692-1698. https:// doi.org/10.1109/BIBM47256.2019.8983088

Publisher's Note Springer Nature remains neutral with regard to jurisdictional claims in published maps and institutional affiliations. 